\title{
The role of partial mediated slip during quasi-static deformation of 3D nanocrystalline metals
}

\author{
Lei Cao ${ }^{1}$, Abigail Hunter ${ }^{2}$, Irene J. Beyerlein ${ }^{3}$ and Marisol Koslowski ${ }^{1}$ \\ ${ }^{1}$ School of Mechanical Engineering, Purdue University, West Lafayette, IN 47907, USA \\ ${ }^{2} X$-Computational Division, Los Alamos National Laboratory, Los Alamos, NM 87545, USA \\ ${ }^{3}$ Theoretical Division, Los Alamos National Laboratory, Los Alamos, NM 87545, USA
}

\begin{abstract}
We present dislocation simulations involving the collective behavior of partials and extended full dislocations in nanocrystalline materials. While atomistic simulations have shown the importance of including partial dislocations in high strain rate simulations, the behavior of partial dislocations in complex geometries with lower strain rates has not been explored. To account for the dissociation of dislocations into partials we include the full representation of the gamma surface for two materials: $\mathrm{Ni}$ and Al. During loading, dislocation loops are emitted from grain boundaries and expand into the grain interiors to carry the strain. In agreement with high strain rate simulations we find that $\mathrm{Al}$ has a higher density of extended full dislocations with smaller stacking fault widths than Ni. We also observe that configurations with smaller average grain size have a higher density of partial dislocations, but contrary to simplified analytical models we do not find a critical grain size below which there is only partial dislocation-mediated deformation. Our results show that the density of partial dislocations is stable in agreement with in situ X-ray experiments that show no increase of the stacking fault density in deformed nanocrystalline Ni. Furthermore, the ratio between partial and extended full dislocation contribution to strain varies with the amount of deformation. The contribution of extended full dislocations to strain grows beyond the contribution of partial dislocations as the deformation proceeds, suggesting that there is no well-defined transition from full dislocation- to partial dislocation-mediated plasticity based uniquely on the grain size.
\end{abstract}

Keywords: Phase field model, Partial dislocations, Stacking fault, Polycrystalline materials, Plasticity

\section{Introduction}

Nanocrystalline (nc) materials and metallic nano layers have received extensive research attention over the last few decades due to their superior mechanical properties [1,2,3,4]. There is general consensus that grain boundaries (GBs) and interfaces play a prominent role in deformation behavior, including strength, hardening and failure. These interfaces act as sources, sinks and barriers for dislocations leading to the Hall-Petch relationship [5, 6], which states that the yield strength is inversely proportional to the square root of the grain size. When the average grain size is in the 10-40 nm range there is a transition to an inverse Hall-Petch effect in which the yield stress decreases with grain refinement. This transition is also attributed to GB-mediated deformation mechanisms. Initially, this transition was linked only to the average grain size, while now simulations have shown that the GB energetics also plays a fundamental role. High-energy GBs are able to accommodate deformation by GB sliding and grain agglomeration [7], while in materials with lower energy GBs, dislocation mechanisms are relatively more important $[8,9,10]$.

Currently, there is no such consensus on the nucleation and propagation of partial dislocations from GBs and their impact on deformation twinning and hardening. While strain-hardening arises from a loss of dislocation mobility due to the interaction with the forest dislocations in coarse-grained polycrystals and single crystals, hardening in nc metals has been linked to the intrinsic stacking fault energy, $\gamma_{s f}$. Yamakov et al. [11] observed in atomistic simulations that in materials with high $\gamma_{s f}$, such as Al, a trailing partial is emitted after the leading partial, forming an extended full dislocation. Conversely, in materials with low $\gamma_{s f}$, like $\mathrm{Au}$ and $\mathrm{Ag}$, the trailing partial does not nucleate and the stacking fault left behind by the leading partial extends over the entire grain. The increase in hardening is attributed to the lack of emission of trailing partials in the latter case. 
Transmission electron microscopy (TEM) experiments by Chen et al. [12] confirmed the presence of deformation twinning created by the nucleation of leading partial dislocations from GBs in nc Al. In their work [12] stacking faults are explained using a line tension approximation by comparing the critical shear stress needed to nucleate a dislocation and the corresponding critical length with the average grain size. Yamakov et al. [11] also suggest that the critical size criterion is related to the stacking fault width relative to the grain size. Chen et al. and Yamakov et al. reported that the transition from full dislocation dominated- to partial dislocation dominated deformation occurs at a critical grain size that is inversely proportional to the $\gamma_{s f}$. Recent experiments in nc Pd [13] observed extensive full dislocation activity at large applied strain. In addition, recent molecular dynamics (MD) and dislocation dynamics simulations suggest that assuming a dependency on $\gamma_{s f}$ may not be enough $[14,15,16]$. The ability to nucleate the trailing dislocation to form an extended partial depends on the unstable stacking fault energy $\left(\gamma_{u s f}\right)$, the applied strain, and the strain rate.

In this paper we perform quasi-static simulations of the evolution of dislocations using a phase field dislocation dynamics (PFDD) approach [17]. We focus on the nucleation and evolution of partial dislocations in grain structures with grain sizes in the range of 10-50 nm. To this end, we incorporate the material gamma surface [18] calculated with atomistic simulations [19] into the PFDD model to account for dislocations dissociating into partials in $\mathrm{Al}$ and Ni. GB mediated deformation mechanisms, such as grain boundary sliding [8] that become of importance in nc structures with grain sizes below $10 \mathrm{~nm}$ or at high temperatures are not included in the present work. The comparison of $\mathrm{Al}$ with $\mathrm{Ni}$ shows that the gamma surface has a large impact on dislocation behavior. The high $\gamma_{s f} / \mu b$ in Al enforces the stacking fault ribbon between the leading and trailing partials to be narrower than in $\mathrm{Ni}$. The grain size influences the relative density of partial and extended full dislocations with initially more extended full dislocations in grain structures with larger grain size. Our simulations show that the contribution to strain of extended full dislocations grows beyond the contribution of partial dislocations at higher applied strain. This tendency was observed in atomistic simulations that show that the density of partial dislocations reaches a plateau [9] or decreases[20] while the density of extended full dislocations increases monotonically.

This paper is organized into the following sections. Section 2 describes the formulation of the PFDD model including the implementation of the gamma surface, the strain-controlled loading condition and the GB structure. In Section 3, we apply our method to investigate effects of the gamma surface, grain size and strain rate on dislocation behavior. In Section 4, we present a summary and concluding remarks.

\section{Numerical method}

In the PFDD approach, dislocations are represented by a set of phase field variables $\xi^{\alpha}(\boldsymbol{r})$. Each phase field is assigned to one of the 12 slip systems, $\alpha$, in face-centered cubic (fcc) metals. An integer jump on the value of $\xi^{\alpha}(\boldsymbol{r})$ in the slip plane indicates the location of a dislocation. The plastic strain $\epsilon_{i j}^{p}(\boldsymbol{r})$ is related to the phase fields through [17]

$$
\epsilon_{i j}^{p}(\boldsymbol{r})=\sum_{\alpha}^{12} \frac{1}{2} \xi^{\alpha}(\boldsymbol{r})\left(b_{i}^{\alpha} m_{j}^{\alpha}+b_{j}^{\alpha} m_{i}^{\alpha}\right) \delta_{\alpha},
$$

where $\boldsymbol{m}^{\alpha}$ is the slip plane normal, $\boldsymbol{b}^{\alpha}$ is the Burgers vector and $\delta_{\alpha}$ is a Dirac delta function confining the slip on the slip plane. The phase fields can be related to local and average quantities such as strain, stress and dislocation density. The local strain can be calculated using the isotropic elastic Green's function, $G(\boldsymbol{r}),[17,21]$ as

$$
\epsilon_{i j}(\boldsymbol{r})=\bar{\epsilon}_{i j}-C_{k l m n} \epsilon_{m n, l}^{p}(\boldsymbol{r}) * \frac{1}{2}\left(G_{i k, j}(\boldsymbol{r})+G_{j k, i}(\boldsymbol{r})\right),
$$

where $\bar{\epsilon}$ is the homogeneous part of the strain, $C_{k l m n}$ are the components of the stiffness tensor and $*$ represents convolution operator. In strain-controlled deformation, $\bar{\epsilon}$ equals the applied strain $\epsilon^{\text {applied }}$, while in stress-controlled deformation $\bar{\epsilon}$ follows [22]

$$
\bar{\epsilon}_{i j}=\frac{1}{V} \int_{V} \epsilon_{i j}^{p}(\boldsymbol{r}) d^{3} r+S_{i j k l} \sigma_{k l}^{\text {applied }},
$$

where the $V$ is the volume of the grain structure and $S_{i j k l}$ are the components of the compliance tensor. The stress and the dislocation density can be calculated as

$$
\sigma_{i j}(\boldsymbol{r})=C_{i j k l}\left(\epsilon_{k l}(\boldsymbol{r})-\epsilon_{k l}^{p}(\boldsymbol{r})\right)
$$




$$
\rho=\frac{1}{V} \sum_{\alpha} \int_{S_{\alpha}}\left|\nabla_{\alpha} \xi^{\alpha}(\boldsymbol{r})\right| d^{2} r,
$$

where $\nabla_{\alpha}$ represents the gradient on the slip system $\alpha$. The evolution of phase fields follows a time-dependent Ginzburg-Landau equation,

$$
\frac{\partial \xi^{\alpha}(\boldsymbol{r})}{\partial t}=-L \frac{\delta E\left[\xi^{1}, \ldots, \xi^{12}\right]}{\delta \xi^{\alpha}(\boldsymbol{r})},
$$

where $L$ is a kinetic coefficient that determines dislocation velocity [19]. Under quasi-static loading the right hand size of Eq. 6 is set to zero such that the simulation allows the system to reach equilibrium, which corresponds to an infinitely long relaxation time or $\dot{\epsilon}=0$.

The system energy $E$ contains elastic interactions among dislocations and the interactions with the applied stress or strain fields, $E^{\text {int }}$, and the misfit energy, $E^{\text {misfit }}$. $E^{\text {misfit }}$ accounts for the energy to create stacking faults [23,24] and is calculated through the gamma surface. The interaction energy is

$$
E^{i n t}=\frac{1}{2} \int_{V} C_{i j k l}\left(\epsilon_{i j}(\boldsymbol{r})-\epsilon_{i j}^{p}(\boldsymbol{r})\right)\left(\epsilon_{k l}(\boldsymbol{r})-\epsilon_{k l}^{p}(\boldsymbol{r})\right) d^{3} r,
$$

where the integration is taken over the entire grain structure. Applying Eq. 3 to Eq. 7 we obtain

$$
\begin{aligned}
E^{i n t} & =\frac{V}{2} C_{i j k l} \bar{\epsilon}_{i j} \bar{\epsilon}_{k l}-C_{i j k l} \bar{\epsilon}_{i j} \sum_{\alpha} \int \xi^{\alpha}(r) b_{k}^{\alpha} m_{l}^{\alpha} d^{3} r \\
& +\frac{1}{2} \int \frac{d^{3} k}{(2 \pi)^{3}} \hat{A}_{i j k l}(\boldsymbol{k}) \sum_{\alpha} \hat{\xi}^{\alpha}(\boldsymbol{k}) b_{i}^{\alpha} m_{j}^{\alpha} \sum_{\alpha^{\prime}} \hat{\xi}^{\alpha^{\prime *}}(\boldsymbol{k}) b_{k}^{\alpha^{\prime}} m_{l}^{\alpha^{\prime}},
\end{aligned}
$$

where $^{\wedge}$ denotes the Fourier transform of the quantity, $\boldsymbol{k}$ is the wave number vector in Fourier space, and

$$
\hat{A}_{i j k l}(\boldsymbol{k})=C_{i j k l}-C_{i j u v} C_{m n k l} \hat{G}_{u m}(\boldsymbol{k}) k_{v} k_{n} .
$$

The model takes into account the nucleation and motion of partial dislocations by including the full gamma surface. This surface is calculated with density functional theory (DFT) or MD simulations of two semi-infinite crystal halves that are rigidly displaced along the glide plane $[19,18,25]$. The planar energy density function, $\phi_{n}\left(\xi^{1}, \xi^{2}, \xi^{3}\right)$, that describes this parameterization for one slip plane $n$ (3 slip systems) has the form $[15,25,26]$

$$
\begin{aligned}
\phi_{n}\left(\xi^{1}, \xi^{2}, \xi^{3}\right)= & \left\{c_{0}+c_{1}\left[\cos 2 \pi\left(\xi^{1}-\xi^{2}\right)+\cos 2 \pi\left(\xi^{2}-\xi^{3}\right)+\cos 2 \pi\left(\xi^{3}-\xi^{1}\right)\right]\right. \\
& +c_{2}\left[\cos 2 \pi\left(2 \xi^{1}-\xi^{2}-\xi^{3}\right)+\cos 2 \pi\left(2 \xi^{2}-\xi^{3}-\xi^{1}\right)+\cos 2 \pi\left(2 \xi^{3}-\xi^{1}-\xi^{2}\right)\right] \\
& +c_{3}\left[\cos 4 \pi\left(\xi^{1}-\xi^{2}\right)+\cos 4 \pi\left(\xi^{2}-\xi^{3}\right)+\cos 4 \pi\left(\xi^{3}-\xi^{1}\right)\right] \\
& +c_{4}\left[\cos 4 \pi\left(3 \xi^{1}-\xi^{2}-2 \xi^{3}\right)+\cos 4 \pi\left(3 \xi^{1}-2 \xi^{3}-\xi^{3}\right)+\cos 4 \pi\left(3 \xi^{2}-\xi^{3}-2 \xi^{1}\right)\right. \\
& \left.+\cos 4 \pi\left(3 \xi^{2}-2 \xi^{3}-\xi^{1}\right)+\cos 4 \pi\left(3 \xi^{3}-\xi^{1}-2 \xi^{2}\right)+\cos 4 \pi\left(3 \xi^{3}-2 \xi^{1}-\xi^{2}\right)\right] \\
& +a_{1}\left[\sin 2 \pi\left(\xi^{1}-\xi^{2}\right)+\sin 2 \pi\left(\xi^{2}-\xi^{3}\right)+\sin 2 \pi\left(\xi^{3}-\xi^{1}\right)\right] \\
& \left.+a_{3}\left[\sin 4 \pi\left(\xi^{1}-\xi^{2}\right)+\sin 4 \pi\left(\xi^{2}-\xi^{3}\right)+\sin 4 \pi\left(\xi^{3}-\xi^{1}\right)\right]\right\} .
\end{aligned}
$$

The material-dependent coefficients, $c_{0}-c_{4}, a_{1}, a_{3}$, are determined by fitting the gamma surface obtained with atomisitc simulations. Details on the fitting procedure can be found in references [16, 25, 27, 28]. Note that Eq. 10 relies on linear combinations of the three active phase field variables on one slip plane. In other words, partial dislocation directions are modeled through the linear combinations of the full dislocation directions in the PFDD model. Because of Eq. 10, the PFDD model accounts for key features in the gamma surface, specifically the local energy maximum and minimum in the[11 2$]$ directions and in the [110] directions as seen in Fig. 1. Rather than depending on a single parameter, such as $\gamma_{s f}$, as in many other models [17, 29, 30, 31,32,33], the present model accounts for the entire material dependent gamma surface. 
Partial dislocations are key in the formation of alternative deformation mechanisms active on the nanoscale, such as twinning [34, 35]. It is expected that changes in deformation processes and appearance of alternative deformation mechanisms due to the presence of partial dislocations and stacking faults will significantly impact the overall mechanical behavior. For such studies, the misfit energy is a key energy term for accurately accounting for mesoscale deformation behavior and partial dislocation behavior. In the present work, the misfit energy that is incorporated into the total energy of the system is obtained by integrating the planar energy density functional in Eq. 10 over one slip plane and then summing over all the slip planes, $n$ :

$$
E^{\text {mistfit }}=\sum_{n} \int \phi_{n}\left(\xi^{1}, \xi^{2}, \xi^{3}\right) d^{2} r .
$$

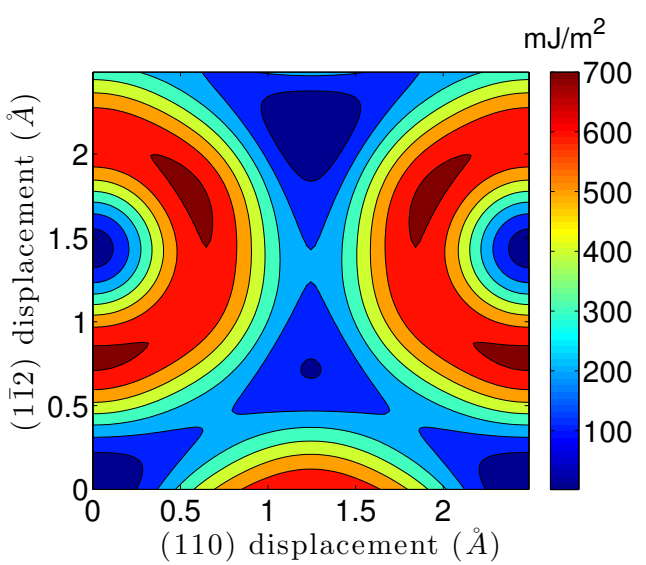

Figure 1: Gamma surface for Ni [15] fitted using Eq.10.

While Eq. 10 fits the full gamma surface, dislocation dynamics methods in general consider only full dislocation nucleation and motion with a simplified planar energy density function of the form [17, 29, 30, 31, 32, 33]

$$
\phi_{n}\left(\xi^{1}, \xi^{2}, \xi^{3}\right)=\sum_{\alpha=1}^{3} A \sin ^{2}\left(\pi \xi^{\alpha}\right),
$$

where the coefficient $A$ is found by fitting the gamma surface in the [110] direction only[19].

\subsection{Polycrystalline structures}

Polycrystalline structures with periodic boundary conditions are used for different grain size simulations. One of these polycrystalline structures is shown in Fig. 2. These strucftures are constructed from the coordinates of atoms [36] built with a Voronoi algorithm [37] as follows. The coordination number of each atom is calculated and non-12coordinated atoms are regarded as GBs. The resulting atomistic GBs are then mapped onto grids used in the PFDD simulations. With this procedure the GBs, colored in blue in Fig. 2, have a finite volume, i.e., are not represented by surfaces as in previous simulations [23].The grid size in the PFDD simulations is set equal to the Burgers vector; therefore, simulations for $\mathrm{Al}$ and $\mathrm{Ni}$ with identical grain structure have a slightly different grain size.

Grain misorientations are accommodated by dislocation networks at GBs [29, 38], in which the structure of these dislocations networks is determined by the type of GB and the misorientation angle. In this work we assign an initial dislocation structure to each GB. Dislocations sliding in the grain interact with GB dislocations by emission of new dislocations, annihilation, transmission and acting as barriers to dislocation motion. 


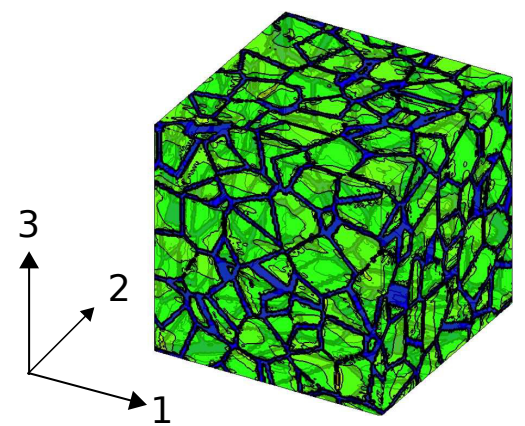

Figure 2: One of the 3D polycrystalline structures used in the PFDD simulations, green regions are grains and blue regions are grain boundaries.

\section{Results}

In this section we present the dislocation dynamics simulations of $\mathrm{nc} \mathrm{Ni}$ and $\mathrm{Al}$ with average grain sizes in the range of 10-50 nm. Table 1 shows the elastic constants, $\gamma_{s f}, \gamma_{u s f}$, the lattice parameter and the Burgers vector of perfect and partial dislocations of $\mathrm{Al}$ and $\mathrm{Ni}$ used in the simulations. By including the gamma surface in Eq. 10 into the misfit energy functional, the dissociation of a perfect dislocation into two Shockley partials bounding a stacking fault is captured.

\begin{tabular}{cccccccc}
\hline & $\mu(\mathrm{GPa})$ & $E(\mathrm{GPa})$ & $\gamma_{s f}\left(\frac{\mathrm{mJ}}{\mathrm{m}^{2}}\right)$ & $\gamma_{u s f}\left(\frac{\mathrm{mJ}}{\mathrm{m}^{2}}\right)$ & $a(\mathrm{~nm})$ & $b(\mathrm{~nm})$ & $b_{p}(\mathrm{~nm})$ \\
\hline $\mathrm{Al}$ & 26.0 & 70.0 & 141.78 & 172.3 & 0.405 & 0.286 & 0.165 \\
$\mathrm{Ni}$ & 75.0 & 200.0 & 84.72 & 211.69 & 0.352 & 0.249 & 0.144 \\
\hline
\end{tabular}

Table 1: Material properties of $\mathrm{Al}$ and $\mathrm{Ni}$ used in the PFDD simulations $[19,26]$.

\subsection{Stress-strain behavior}

Fig. 3 (a) compares the stress-strain curves for nc Ni under stress-controlled and strain-controlled deformation using the full parametrization of the gamma surface, Eq. 10. Under stress-controlled conditions a shear stress $\sigma_{13}$ is applied (see Fig. 2 for axis directions). During strain-controlled simulations the applied strain has a component $\epsilon_{13}$. Both curves are simulated under quasi-static loading conditions but have very different features. In the straincontrolled case, the nucleation and propagation of dislocations lead to an abrupt stress drop after yielding. While in the stress-controlled case the nucleation of dislocations causes a strain burst.

In Fig. 3 (b) we emphasize the importance of using the parametrization of the full gamma surface. Fig. 3 (b) displays the stress-strain curves for $d=19.2 \mathrm{~nm} \mathrm{Ni}$ obtained using the parametrization of the gamma surface in Eq. 10 and the sinusoidal approximation in Eq. 12 under quasi-static strain-controlled conditions. With the incorporation of the entire gamma surface into the energetics of the system, the material selects to nucleate leading partial dislocations followed by trailing partials. Clearly when using the expression in Eq. 12, only perfect dislocations are nucleated in the system, raising the energy needed to activate and glide a dislocation and, consequently, increasing the yield stress. All the simulations that follow are performed using the full parametrization of the gamma surface.

Figure 4 shows quasi-static stress-strain curves for different grain structures with grain sizes ranging from 10 to $40 \mathrm{~nm}$ for $\mathrm{nc} \mathrm{Ni}$. As expected, the simulations can capture the Hall-Petch effect for the range of microstructures simulated. Plastic deformation is carried out by dislocation glide and therefore, the Hall-Petch effect is caused by the confinement of dislocations in grains. We included GB mediated deformation mechanisms in past simulations that shows a transition to inverse Hall-Petch effect for grain sizes smaller than $16 \mathrm{~nm}$ [8]. These mechanisms are not included here and therefore, this transition is not present. We observe a considerable amount of hardening after yielding in Fig. 4. By not allowing GB-mediated mechanisms to accommodate the deformation we obtain large dislocation densities that increase the rate of hardening. 


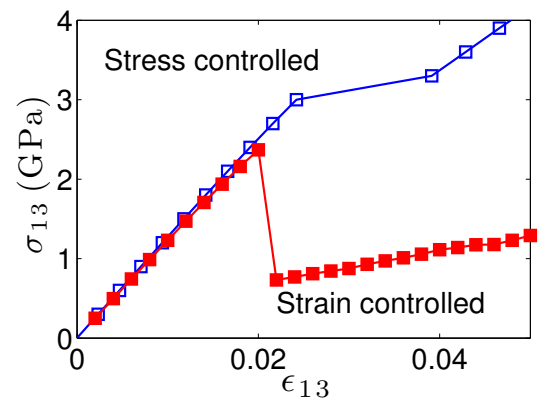

(a) $d=40 \mathrm{~nm}$

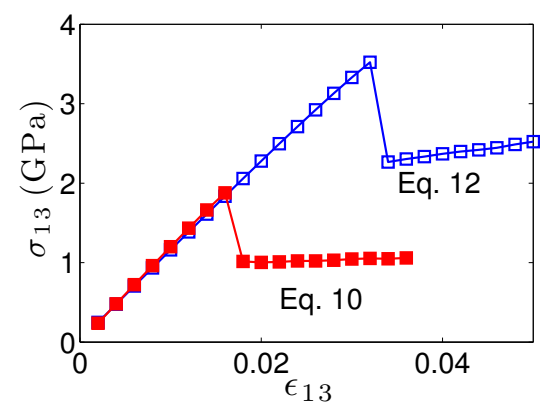

(b) $d=19.2 \mathrm{~nm}$

Figure 3: Stress-strain curves for Ni (a) in stress- and strain-controlled deformation using Eq. 10; (b) with different parameterizations of the gamma surface under strain-controlled deformation.

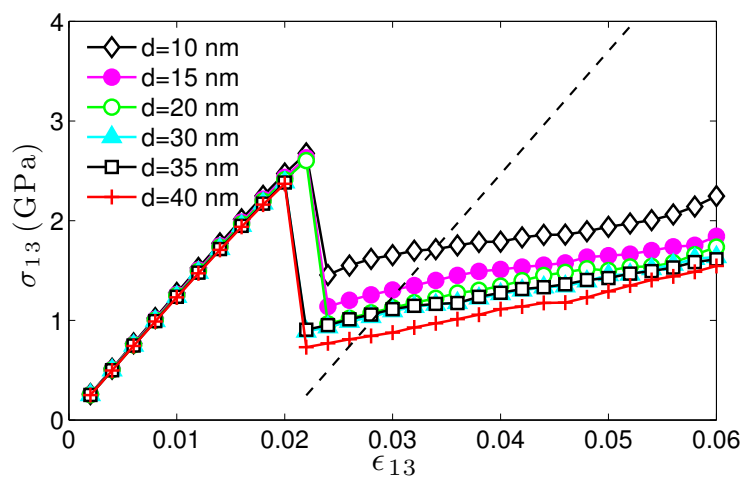

Figure 4: Simulated stress-strain curves for $\mathrm{nc}$ Ni with grain sizes ranging from $10 \mathrm{~nm}$ to $40 \mathrm{~nm}$.

\subsection{Nucleation of partial dislocations}

Simulations in $\mathrm{Ni}$ and $\mathrm{Al}$ are used to compare the effect of large (Al) and small (Ni) stacking fault energies on the nucleation and propagation of partial dislocations followed by extended full dislocations. In particular, we conduct PFDD simulations on $\mathrm{Ni}$ and $\mathrm{Al}$ with identical grain structures under strain-controlled loading conditions. The simulated stress-strain curves for $\mathrm{Ni}$ and $\mathrm{Al}$ are shown in Fig. 5 under quasi-static loading.

In Eq. 10, the gamma surface describes the penalty for creating a stacking fault as the dislocation ensemble evolves. One of the components of the Burgers vector of the two partial dislocations have like sign and therefore, repel each other. However, due to the energy penalty associated with the creation of a stacking fault, the two partial dislocations may remain close with a narrow stacking fault region. In that case, the leading and trailing partials move together and are considered an extended full dislocation. The equilibrium stacking fault widths in simple configurations have been calculated with the PFDD method for several fcc metals and the results have shown good agreement with available experimental equilibrium widths $[15,28]$. The energy per unit area in the gamma surface is parametrized by the combination of phase fields $\xi^{\alpha}(\boldsymbol{r})$ that represent displacements in the $<110>$ directions on the slip planes (111). Accordingly, the sum of the phase fields, $\Delta$ represents the amount of slip on the slip plane [39] and is given by

$$
\boldsymbol{\Delta}=\sum_{\alpha=1}^{3} \xi^{\alpha}(\boldsymbol{r}) \boldsymbol{b}^{\alpha}
$$

Unslipped regions in the slip plane have zero displacement and regions slipped by full dislocations have displace- 


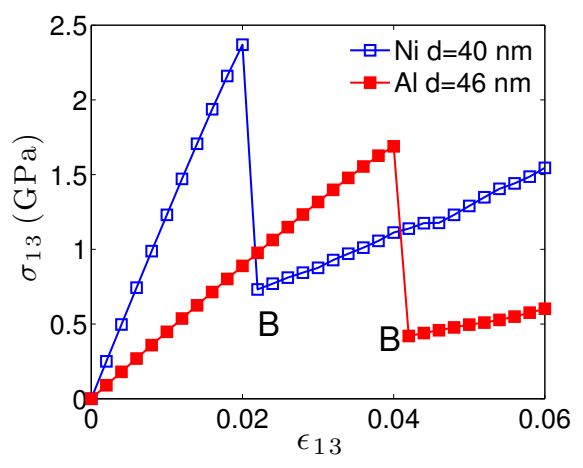

Figure 5: Simulated stress-strain curves for nc Ni and nc Al.

ments in the direction of the Burgers vector of a perfect dislocation, $<110>$. Regions slipped by a partial dislocation have a displacement in the $<211>$ direction resulting in a stacking fault. Therefore, to identify stacking faults, we define the projection of the total displacement $\Delta$ in the [11 2 ] direction:

$$
\Delta_{1}=\Delta \cdot[1 \overline{1} 2]
$$

Fig. 6 (a-d) shows contours of $\Delta_{1}$, yellow areas represent stacking faults and have a displacement $\boldsymbol{\Delta}=\frac{1}{6}$ [211]; green lines represent the two Shockley partials; blue regions are GBs. This figure shows the sequence of the nucleation of a leading partial followed by a stacking fault and the trailing partial in a grain and the transmission across a GB. This visualization scheme is similar to the common neighbor analysis [40] that is commonly used to visualize stacking faults, dislocations and grain boundaries in MD simulations. For comparison we show a similar process simulated with molecular dynamics from Reference [14] in Fig. 6 (e-h). In Fig. 7 unslipped regions and regions slipped by a displacement equal to a perfect dislocation in the slip plane have $\Delta_{1}=0$. Because in both cases these regions have perfect crystalline structure they are assigned white color.

The simulations predict the nucleation of leading partials from GBs followed by trailing partials, forming extended full dislocations as the applied strain is increased. The sequence shown in Fig. 7 (a-c) displays the details of the dislocation nucleation process in $\mathrm{Al}$ in response to an applied strain $\epsilon_{13}=0.04$. The same nucleation process in $\mathrm{Ni}$ at $\epsilon_{13}=0.02$ is shown in Fig. 7 (d-f). In both materials a leading partial is nucleated from the GB and the yellow area is the stacking fault left behind by the leading partial. In Fig. 7(b), the trailing partial has nucleated and removes part of the stacking fault defect. Afterwards the two partials form an extended full dislocation that glides as a pair.

\subsection{Material and loading conditions effects}

To examine the microstructural details of the nucleation and gliding process in several grains, cross sections from the 3D grain structures for $\mathrm{Al}$ with $d=46 \mathrm{~nm}$ and $\mathrm{Ni}$ with $d=40 \mathrm{~nm}$ are displayed in Fig. 8 (a) and (b). These structures correspond to the configurations after the stress drop labeled B in Fig. 5. The comparison of this process in different materials reveals that the stacking fault ribbon is narrower in $\mathrm{Al}$ than in $\mathrm{Ni}$. This is expected because the width of the stacking fault is inversely proportional to $\frac{\gamma_{s f}}{\mu b}[15,16,41]$.

The areas slipped by partial dislocations, $A_{p}$, and by extended full dislocations, $A_{f}$, are quantified for different applied strain, strain rates and grain size. In Fig. 9 (a) and (b) $A_{p}$ and $A_{f}$ are plotted as a function of the applied strain for $\mathrm{nc} \mathrm{Al}$ and nc Ni during quasi-static deformation. For both $\mathrm{Al}$ and $\mathrm{Ni} A_{f}$ increases with the applied strain while $A_{p}$ remains approximately constant. Leading partial dislocations count towards $A_{p}$, after the trailing partials are nucleated, the area slipped by an extended full dislocation will be added to $A_{f}$. The balance between the addition and the subtraction processes results in a stable $A_{p}$ in $\mathrm{Ni}$. In $\mathrm{Al}$ the nucleation of the trailing partial has a lower energy barrier [41] and therefore, the amount of extended full dislocations surpasses the nucleation of new leading partials, leading to a moderate decrease of $A_{p}$ and an increase of $A_{f}$. 


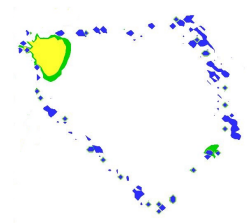

(a)

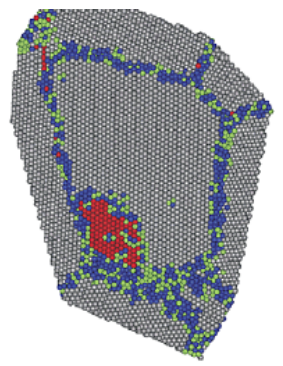

(e)

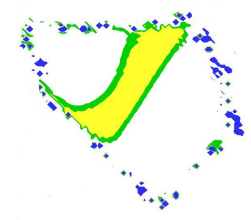

(b)

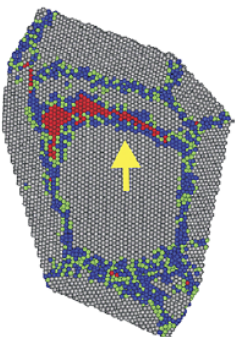

(f)

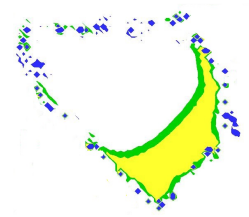

(c)

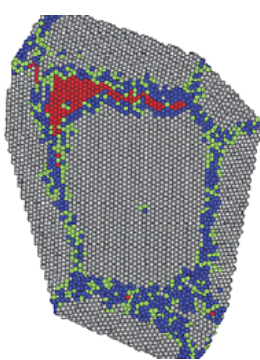

(g)

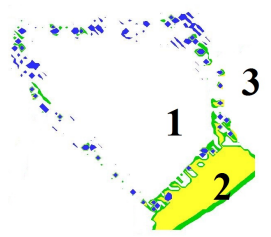

(d)

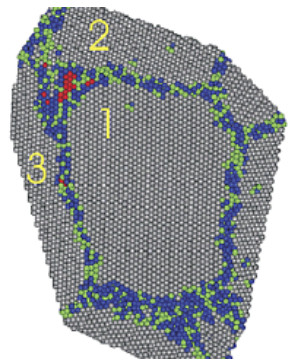

(h)

Figure 6: Evolution of dislocations nucleated at GBs and formation of stacking faults in (a-d) with PFDD simulations in nc Ni with d=15nm. Yellow areas are stacking faults, green lines represent Shockley partials, and blue regions are GBs. 1,2 and 3 denote different grains. (e-h) Molecular dynamics simulations in $\mathrm{nc} \mathrm{Al}$ from [14].

A monotonically increasing $A_{f}$ is also observed in MD simulations on $\mathrm{nc} \mathrm{Cu}$ with a grain size $\mathrm{d}=15 \mathrm{~nm}[9,20]$. In these simulations the contribution to strain of partial dislocations is initially larger than the contribution of extended full dislocations, as deformation proceeds $A_{f}$ slightly exceeds $A_{p}$. On the other hand, Tucker et al. [20] observed the onset of the reduction on partial dislocation mediated deformation at a strain $8 \%$ with a strain rate $10^{9} / \mathrm{s}$, while Vo et al. [9] observed a plateau at a strain $20 \%$ with a strain rate $10^{10} / \mathrm{s}$. This behavior is not observed in $5 \mathrm{~nm}$ and $10 \mathrm{~nm}$ grain size samples at these strain rates. To study the dependency of this phenomenon on the strain rate we perform PFDD simulations at a strain rate $1.6 \cdot 10^{6} / \mathrm{s}$ [42] for $\mathrm{nc} \mathrm{Ni}$ with grain size $d=40 \mathrm{~nm}$. This strain rate is calculated assuming a dislocation velocity of $2 \mathrm{~m} / \mathrm{s}$ [43]. Figure 9 (c) shows this simulation. Initially, $A_{p}$ is larger than $A_{f}$. At an applied strain of $3 \%, A_{f}$ starts increasing and $A_{p}$ decreases. The strain at which this transition occurs increases with the strain rate, indicating that strain rate as well as GB mechanisms and grain size play a prominent role. Figure 9 (d) presents the ratio of the contribution of partial dislocations to extended full dislocations for cases shown in Figures 9 (a-c). Only at high strain rates the contribution of partial dislocations overpasses the extended full dislocations.

\subsection{Effect of the grain size on the activity of partial dislocations}

Figure 10 shows the dislocation structures for an applied strain $\epsilon_{13}=0.024$ for Ni grain structures with grain sizes $d=10,15$, and $30 \mathrm{~nm}$. It is important to notice that while we see dislocation activities in all the grains for $\mathrm{d}=40 \mathrm{~nm}$, shown in Fig. 8(b), the dislocation activity in smaller grain size samples occurs only in a limited number of grains as shown in Fig. 10. The $30 \mathrm{~nm}$ and $40 \mathrm{~nm}$ grain size structures have a higher amount of extended full dislocations than the smaller grain size.

It was proposed previously that at a critical grain size there is a transition from full dislocation-dominated plasticity to partial dislocation-dominated plasticity. Chen et al. [12] studied this transition using the classical line tension model by comparing the critical stress to nucleate a perfect dislocation and a partial dislocation. The critical grain size is

$$
d_{c}=\frac{2 \alpha \mu\left(b-b_{p}\right) b_{p}}{\gamma_{s f}}
$$

where $b$ and $b_{p}$ are magnitudes of the Burgers vector of full and partial dislocations and $\alpha$ is a parameter between 0.5 and 1 that reflects the edge/screw character of the dislocation. With the constants in Table 1 we find $d_{c}=17-35 \mathrm{~nm}$ for $\mathrm{Ni}$ and $d_{c}=8-16 \mathrm{~nm}$ for $\mathrm{Al}$. 


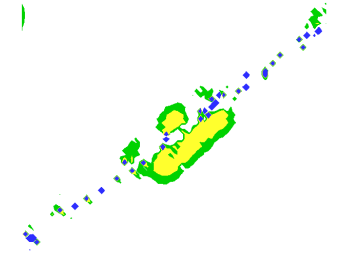

(a)

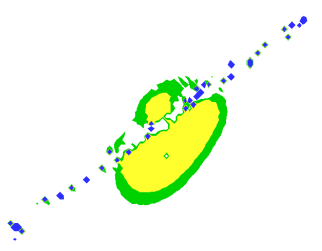

(d)

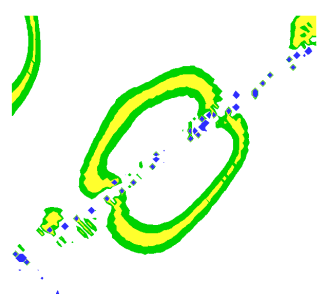

(b)

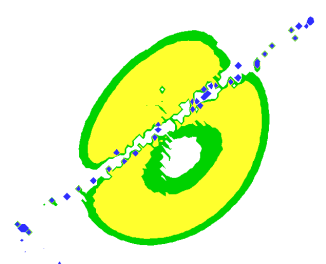

(e)

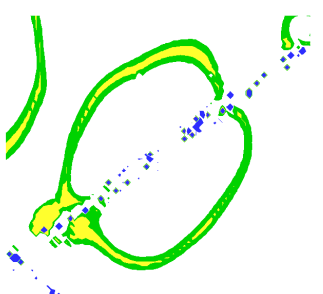

(c)

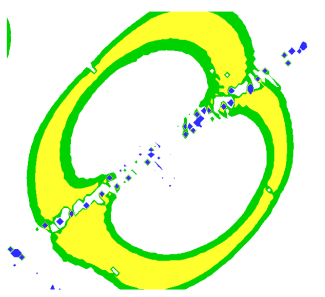

(f)

Figure 7: Snapshots of dislocation nucleation process in $\mathrm{Al}(\mathrm{a}-\mathrm{c})$ and in $\mathrm{Ni}$ (d-f).

In the above model the transition from extended full dislocation- to partial dislocation-dominated deformation in nc metals only depends on the grain size. It is important to note that this approximation does not take into account the current state of deformation, i.e. strain level. Another model presented by Yamakov et al. [11] introduces a dislocation splitting distance related to the applied stress. Yamakov et al. [11] suggested that the critical grain size, $d_{c}$, is:

$$
d_{c}=\frac{K b_{p}^{2}}{\gamma_{s f}\left(1-\tau / \tau_{\infty}\right)}
$$

where $\tau$ is the resolved shear stress in the plane of the dislocations and $\tau_{\infty}$ is the resolved shear stress at which the distance between partial dislocations is infinitely large. This model predicts that the critical grain size below which only partial dislocation mediated deformation is relevant increases with the applied stress. However, it is important to notice that the models in Eqs 15 and 16 do not take into account the nucleation of the trailing partial dislocations, but just compare the stacking fault width with the grain size.

The effect of the average grain size on the dislocation activity is evident in the PFDD simulations. Fig. 11 (a) shows how $A_{p} / A_{f}$ decreases with increasing grain size for applied strains $\epsilon_{13}=0.024$ and 0.040 . It is important to notice that the difference between partial and extended full dislocations is more pronounced at smaller grain sizes and at lower applied strains. In Fig. 11(b), $A_{p} / A_{f}$ decreases with increasing strain level, suggesting that the current state of deformation is an important factor on the activity of partial and full dislocations in agreement with Eq. 16. For example, the solid squares in Fig. 11(a) imply that at $\epsilon_{13}=0.024$, there exists a transition from partial- to extended full dislocation-mediated deformation around $d=22 \mathrm{~nm}$. However, the open squares in Fig. 11(a) confirm that at larger deformation $\epsilon_{13}=0.04$, the deformation is dominated by extended full dislocations for all grain sizes explored in the current work.

\section{Summary}

We conducted dislocation dynamics simulations to study the collective behavior of partial and full extended dislocations in nc materials under strain-controlled loading conditions. By incorporating the full material gamma surface, we can capture stacking fault defects and activities of partial and full extended dislocations. We observe that leading 


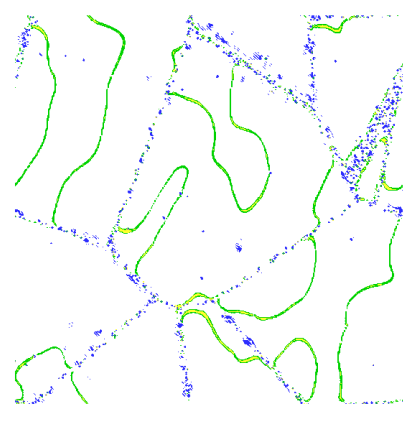

(a) $\mathrm{Al} d=46 \mathrm{~nm}$

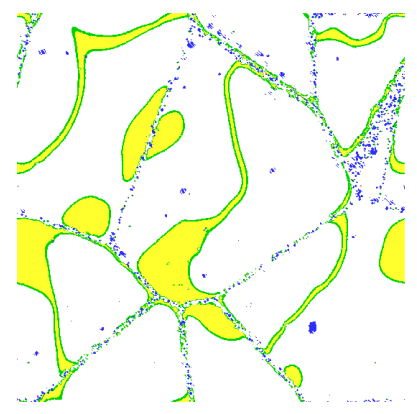

(b) Ni $d=40 \mathrm{~nm}$

Figure 8: Dislocations and stacking fault structures in (a) $\mathrm{Al}$ and (b) Ni.

partial dislocations are nucleated at the GB and expand into the grain interiors, leaving behind a stacking fault defect. Trailing partial dislocations are nucleated at higher applied strains to form extended full dislocations.

Simulations were performed on $\mathrm{nc} \mathrm{Ni}$ and $\mathrm{nc} \mathrm{Al}$ with identical grain structures to identify the effect of the gamma surface. As expected, we observe a lower ratio of partial to extended full dislocations and smaller stacking fault widths in $\mathrm{Al}$ than in $\mathrm{Ni}$ due to the lower energy barrier for the nucleation of the trailing partial dislocation in Al. As the applied strain is increased, trailing partial dislocations are nucleated raising the contribution of extended full dislocations in both materials.

To elucidate the effect of the grain size on the nucleation of dislocations and the evolution of slip, we conducted simulations for $\mathrm{Ni}$ with grain sizes in the range of $10-40 \mathrm{~nm}$. We explicitly calculated the contribution to strain of partial and extended full dislocations over a large range of applied strain and grain sizes. We found that the contribution to strain of partial and extended full dislocations are not only affected by the grain size but also by the state of deformation. Specifically, at the same deformation level, grain structures with smaller grain size have a higher ratio $A_{p} / A_{f}$ as shown in Fig. 11(a); the ratio of partial dislocations to extended full dislocations decreases as the deformation proceeds in all the simulations performed in the current work. In addition, in quasi-static simulations for $\mathrm{d}=40 \mathrm{~nm} \mathrm{Ni}$ we found that the contribution of full dislocations is always higher than that of partial dislocations.

The trend of monotonically increasing contribution of extended full dislocations is also observed in MD simulations on $\mathrm{nc} \mathrm{Cu}$ with a grain size $\mathrm{d}=15 \mathrm{~nm}[9,20]$. In these simulations the contribution to strain of partial dislocations is initially larger that that of extended full dislocations. But as deformation proceeds they observe that $A_{f}$ slightly exceeds $A_{p}$. Our results for finite strain rate simulation on $\mathrm{d}=40 \mathrm{~nm} \mathrm{Ni}$ (Fig. 9(c)) confirm this tendency. Initially the contribution of partial dislocations is higher than that of extended full dislocations, but at an applied strain of 0.035 $A_{f}$ is larger than $A_{p}$. In quasi-static simulations on the contrary, the contribution of extended full dislocations always surpasses the contribution of partial dislocations.

In summary, our simulations show that there is a strong dependency on grain size, state of deformation and strain rate on the predominance of partial over extended full dislocation that simplified models are not able to capture. We also show that results at high strain rates cannot always be extrapolated to experimental conditions at lower strain rates. The nucleation of leading partials prevailing over the nucleation of trailing partials to form extended full dislocations in $15 \mathrm{~nm}$ grain sized samples in MD simulations may be an effect of the high strain rates applied during the simulations.

\section{Acknowledgements}

This work was performed with support from the United States Department of Energy Office of Basic Energy Science(US DOE-BES) under contract No. DE-FG02-07ER46398. We would like to thank Prof. Strachan and Dr. H. Kim for providing us atomistic descriptions of the grain structures used in this paper. 


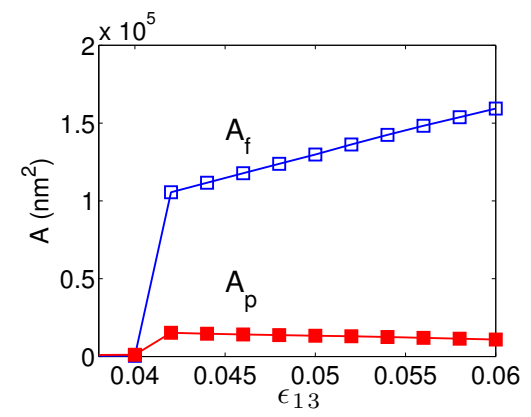

(a)

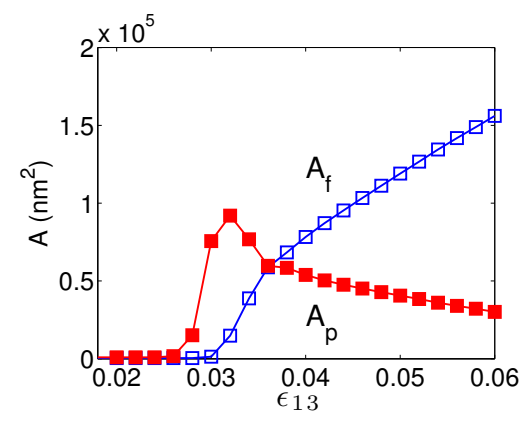

(c)

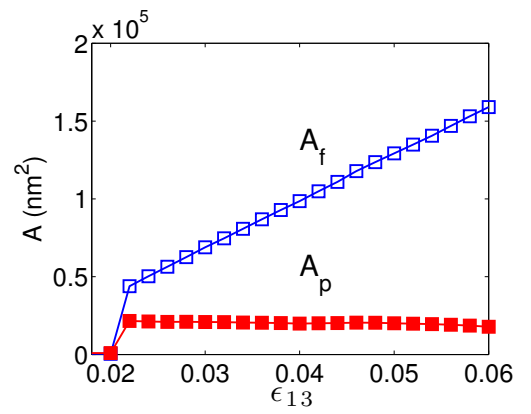

(b)

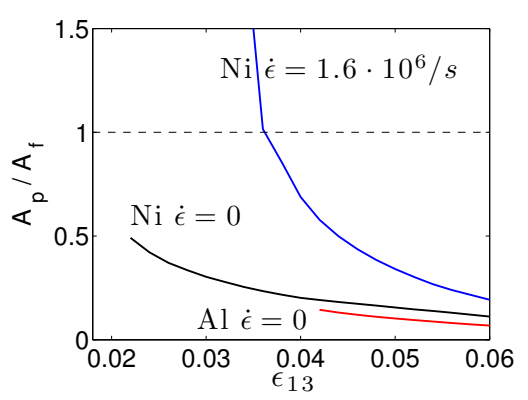

(d)

Figure 9: Dislocation contribution to deformation in (a) nc Al $\dot{\epsilon}=0, d=46 \mathrm{~nm}$; (b) $\mathrm{nc} \mathrm{Ni} \dot{\epsilon}=0, d=40 \mathrm{~nm} ;(\mathrm{c}) \mathrm{nc} \mathrm{Ni} \dot{\epsilon}=1.6 \cdot 10^{6} / s, d=40 \mathrm{~nm}$. (d) $A_{p} / A_{f}$ versus applied strain for $\mathrm{nc} \mathrm{Ni}$ and $\mathrm{Al}$ at different strain rates.

[1] K. Kumar, H. V. Swygenhoven, S. Suresh, Mechanical behavior of nanocrystalline metals and alloys, Acta Materialia 51 (19) (2003) 5743 - 5774, the Golden Jubilee Issue. Selected topics in Materials Science and Engineering: Past, Present and Future. doi:http://dx.doi.org/10.1016/j.actamat.2003.08.032.

URL http://www.sciencedirect.com/science/article/pii/S1359645403005135

[2] D. Wolf, V. Yamakov, S. Phillpot, A. Mukherjee, H. Gleiter, Deformation of nanocrystalline materials by molecular-dynamics simulation: relationship to experiments?, Acta Materialia 53 (1) (2005) 1 - 40. doi:http://dx.doi.org/10.1016/j.actamat.2004.08.045.

URL http://www.sciencedirect.com/science/article/pii/S1359645404005087

[3] M. Meyers, A. Mishra, D. Benson, Mechanical properties of nanocrystalline materials, Progress in Materials Science 51 (4) (2006) 427 556. doi:DOI: 10.1016/j.pmatsci.2005.08.003.

URL http: //www.sciencedirect.com/science/article/pii/S0079642505000447

[4] J. Wang, A. Misra, An overview of interface-dominated deformation mechanisms in metallic multilayers, Current Opinion in Solid State and Materials Science 15 (1) (2011) 20 - 28. doi:http://dx.doi.org/10.1016/j.cossms.2010.09.002.

URL http://www.sciencedirect.com/science/article/pii/S135902861000046X

[5] E. O. Hall, The deformation and ageing of mild steel, Proceedings of the Phys. Society of London 64 (381B) (1951) $747-753$.

[6] N. J. Petch, Cleavage strength of polycrystals, J. Iron Steel Inst. 174 (Part 1) (1953) 25-28.

[7] Z. Shan, E. A. Stach, J. M. K. Wiezorek, J. A. Knapp, D. M. Follstaedt, S. X. Mao, Grain boundary-mediated plasticity in nanocrystalline nickel, Science 305 (5684) (2004) 654-657. arXiv:http://www.sciencemag.org/content/305/5684/654.full.pdf, doi:10.1126/science.1098741. URL http: //www.sciencemag.org/content/305/5684/654. abstract

[8] M. Koslowski, D. W. Lee, L. Lei, Role of grain boundary energetics on the maximum strength of nanocrystalline nickel, Journal of the Mechanics and Physics of Solids 59 (7) (2011) 1427 - 1436. doi:http://dx.doi.org/10.1016/j.jmps.2011.03.011. URL http://www.sciencedirect.com/science/article/pii/S0022509611000627

[9] N. Q. Vo, R. S. Averback, P. Bellon, S. Odunuga, A. Caro, Quantitative description of plastic deformation in nanocrystalline cu: Dislocation glide versus grain boundary sliding, Phys. Rev. B 77 (2008) 134108. doi:10.1103/PhysRevB.77.134108. URL http://link.aps.org/doi/10.1103/PhysRevB.77.134108

[10] R. A. Lebensohn, E. M. Bringa, A. Caro, A viscoplastic micromechanical model for the yield strength of nanocrystalline materials, Acta Materialia, 55 (2007) 261-271.

[11] V. Yamakov, D. Wolf, S. R. Phillpot, A. K. Mukherjee, H. Gleiter, Deformation-mechanism map for nanocrystalline metals by moleculardynamics simulation, Nat Mater 3 (1) (2004) 43-47.

URL http://dx.doi .org/10.1038/nmat1035 


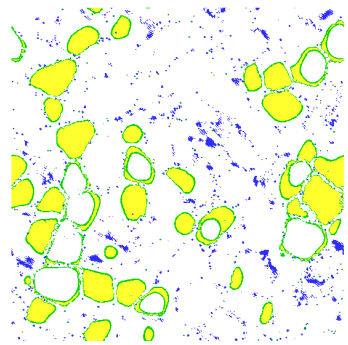

(a) $\mathrm{d}=10 \mathrm{~nm}$

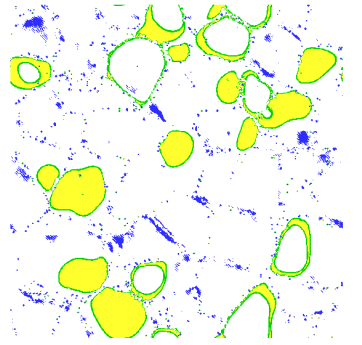

(b) $\mathrm{d}=15 \mathrm{~nm}$

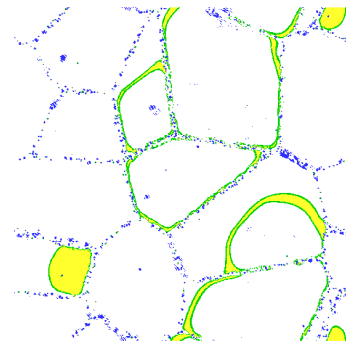

(c) $\mathrm{d}=30 \mathrm{~nm}$

Figure 10: Dislocation patterns on cross sections of nc Ni grain structures at an applied strain of $\epsilon_{13}=0.024$.

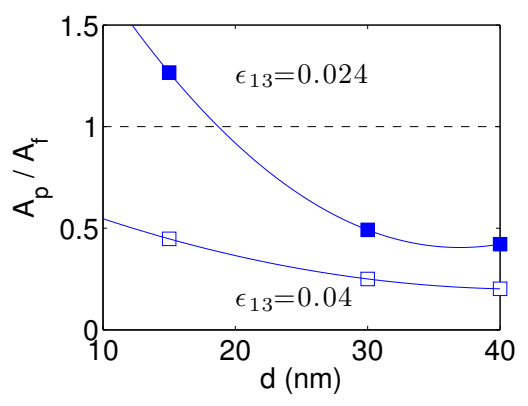

(a)

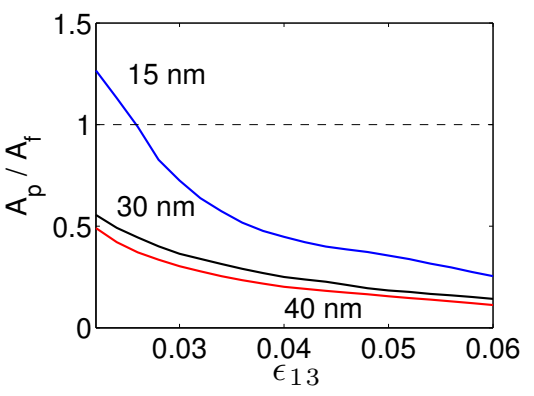

(b)

Figure 11: (a) $A_{p} / A_{f}$ (a) as a function of the grain size for Ni at an applied strain $\epsilon_{13}=0.024$ and 0.040 ; (b) as a function of the applied strain for nc Ni with $\mathrm{d}=15,30$ and $40 \mathrm{~nm}$.

[12] M. Chen, E. Ma, K. J. Hemker, H. Sheng, Y. Wang, X. Cheng, Deformation twinning in nanocrystalline aluminum, Sience 300 (2003) 1275-1277.

[13] Y. Ivanisenko, L. Kurmanaeva, J. Weissmueller, K. Yang, J. Markmann, H. Rösner, T. Scherer, H.-J. Fecht, Deformation mechanisms in nanocrystalline palladium at large strains, Acta Materialia 57 (11) (2009) 3391 - 3401. doi:http://dx.doi.org/10.1016/j.actamat.2009.03.049. URL http://www.sciencedirect.com/science/article/pii/S1359645409002092

[14] A. Frøseth, P. Derlet, H. V. Swygenhoven, Dislocations emitted from nanocrystalline grain boundaries: nucleation and splitting distance, Acta Materialia 52 (20) (2004) $5863-5870$.

[15] A. Hunter, I. J. Beyerlein, T. C. Germann, M. Koslowski, Influence of the stacking fault energy surface on partial dislocations in fcc metals with a three-dimensional phase field dislocations dynamics model, Phys. Rev. B 84 (2011) 144108. doi:10.1103/PhysRevB.84.144108. URL http://link.aps .org/doi/10.1103/PhysRevB.84.144108

[16] A. Hunter, R. F. Zhang, I. J. Beyerlein, T. C. Germann, M. Koslowski, Dependence of equilibrium stacking fault width in fcc metals on the $\gamma$-surface, Modelling and Simulation in Materials Science and Engineering 21 (025015) (2013) 1-19.

[17] M. Koslowski, A. Cuitiño, M. Ortiz, A phase-field theory of dislocations dynamics, strain hardening and hysteresis in ductile single crystals, Journal of the Mechanics and Physics of Solids 50 (12) (2002) 2597-2635.

[18] V. Vitek, Intrinsic stacking faults in body-centered cubic crystals, Philosophical Magazine 18 (154) (1968) 773-786.

[19] D. W. Lee, H. Kim, A. Strachan, M. Koslowski, Effect of core energy on mobility in a continuum dislocation model, Phys. Rev. B 83 (10) (2011) 104101. doi:10.1103/PhysRevB.83.104101.

[20] G. J. Tucker, S. Tiwari, J. A. Zimmerman, D. L. McDowell, Investigating the deformation of nanocrystalline copper with microscale kinematic metrics and molecular dynamics, Journal of the Mechanics and Physics of Solids 60 (3) (2012) 471 - 486. doi:http://dx.doi.org/10.1016/j.jmps.2011.11.007. URL http: //www.sciencedirect.com/science/article/pii/S0022509611002195

[21] T. Mura, Micromechanics of defects in solids, Kluwer Academic Publishers, 1987.

[22] L. Lei, M. Koslowski, Mesoscale modeling of dislocations in molecular crystals, Philosophical Magazine 91 (6) (2011) 865-878. arXiv:http://www.tandfonline.com/doi/pdf/10.1080/14786435.2010.533135, doi:10.1080/14786435.2010.533135.

URL http: //www. tandfonline.com/doi/abs/10.1080/14786435.2010.533135

[23] L. Cao, M. Koslowski, Effect of microstructural uncertainty on the yield stress of nanocrystalline nickel, Acta Materialia 61 (4) (2013) 1413 
- 1420. doi:http://dx.doi.org/10.1016/j.actamat.2012.11.018.

URL http://www.sciencedirect.com/science/article/pii/S1359645412008221

[24] A. Hunter, F. Saied, C. Le, M. Koslowski, Large-scale 3d phase field dislocation dynamics simulations on high-performance architectures, International Journal of High Performance Computing Applicationsdoi:10.1177/1094342010382534.

URL http://hpc.sagepub.com/content/early/2010/11/04/1094342010382534.abstract

[25] G. Schoeck, The core structure, recombination energy and peierls energy for dislocations in al, Philosophical Magazine A 81 (5) (2001) 1161-1176. arXiv:http://www.tandfonline.com/doi/pdf/10.1080/01418610108214434, doi:10.1080/01418610108214434.

URL http: //www.tandfonline.com/doi/abs/10.1080/01418610108214434

[26] C. Shen, Y. Wang, Incorporation of -surface to phase field model of dislocations: simulating dislocation dissociation in fcc crystals, Acta Materialia 52 (3) (2004) 683 - 691. doi:http://dx.doi.org/10.1016/j.actamat.2003.10.014.

URL http://www.sciencedirect.com/science/article/pii/S1359645403006207

[27] A. Hunter, I. Beyerlein, Stacking fault emission from grain boundaries: Material dependencies and grain size effects, Materials Science and Engineering: A 600 (0) (2014) 200 - 210. doi:http://dx.doi.org/10.1016/j.msea.2014.02.030.

URL http://www.sciencedirect.com/science/article/pii/S0921509314001725

[28] A. Hunter, R. F. Zhang, I. J. Beyerlein, The core structure of dislocation and their relationship to the material $\gamma$-surface, Journal of Applied Physics 115 (2014) 134314.

[29] J. P. Hirth, J. Lothe, Theory of Dislocations, McGraw-Hill, New York, 1968.

[30] Y. T. Zhu, X. Z. Liao, S. G. Srinivasan, E. J. Lavernia, Nucleation and deformation twins in nanocrystalline face-centered-cubic metals by severe plastic deformation, Journal of Applied Physics 98 (034319) (2005) 1-8.

[31] Z. Q. Wang, I. J. Beyerlein, Stress orientation and relativistic effects on the separation of moving screw dislocations, Physical Review B 77 (184112) (2008) 1-14.

[32] J. Douin, F. Pettinari-Sturmel, A. Coujou, Dissociated dislocations in confined plasticity, Acta Materialia 55 (2007) 6453-6458.

[33] E. Martinez, J. Marian, A. Arsenlis, M. Victoria, J. Perlado, Atomistically informed dislocation dynamics in fcc crystals, Journal of the Mechanics and Physics of Solids 56 (2008) 869-895.

[34] X. Z. Liao, F. Zhou, E. J. Lavernia, S. G. Srinivasan, M. I. Baskes, D. W. He, Y. T. Zhu, Deformation mechanism in nanocrystalline al: Partial dislocation slip, Applied Physics Letters 83 (4) (2003) 632-634.

[35] Y. T. Zhu, X. Z. Liao, X. L. Wu, Deformation twinning in bulk nano crystalline metals: Experimental observations, Journal of the Minerals, Metals and Materials Society 60 (9) (2008) 60-64.

[36] H. Kim, A. Strachan, Deformation in nanocrystalline metal -comparison of mechanical behavior in a bulk with in corresponding thin slab., submitted to Journal of Applied Physics.

[37] G. Vornoi, J. R. Angew, Nouvelles applications des paramètres continus à la théorie des formes quadratiques., J. Reine Angew. Math. 134.

[38] A. Sutton, R. Balluffi, Interfaces in Crystalline Materials, Oxford University Press, 2006.

[39] M. Koslowski, M. Ortiz, A multi-phase field model of planar dislocation networks, Modelling and Simulation in Materials Science and Engineering 12 (2004) 1087-1097.

[40] J. D. Honeycutt, H. C. Andersen, Molecular dynamics study of melting and freezing of small lennard-jones clusters, The Journal of Physical Chemistry 91 (19) (1987) 4950-4963. arXiv:http://dx.doi.org/10.1021/j100303a014, doi:10.1021/j100303a014. URL http://dx.doi.org/10.1021/j100303a014

[41] H. Van Swygenhoven, P. M. Derlet, A. G. Froseth, Stacking fault energies and slip in nanocrystalline metals, Nat Mater 3 (6) (2004) 403.

[42] L. Cao, M. Koslowski, Achieving nanoscale deformation simulations at experimental strain rate conditions, In preparation.

[43] Y. Fan, Y. Osetsky, S. Yip, B. Yildiz, Onset mechanism of strain-rate-induced flow stress upturn, Phys. Rev. Lett. 109 (2012) 135503. doi:10.1103/PhysRevLett.109.135503. URL http://link.aps.org/doi/10.1103/PhysRevLett.109.135503 